TRANSACTIONS OF THE

AMERICAN MATHEMATICAL SOCIETY

Volume 357, Number 11, Pages 4661-4679

S 0002-9947(05)03814-6

Article electronically published on June 21, 2005

\title{
OUTER FACTORIZATIONS IN ONE AND SEVERAL VARIABLES
}

\author{
MICHAEL A. DRITSCHEL AND HUGO J. WOERDEMAN
}

In memory of Marvin Rosenblum

\begin{abstract}
A multivariate version of Rosenblum's Fejér-Riesz theorem on outer factorization of trigonometric polynomials with operator coefficients is considered. Due to a simplification of the proof of the single variable case, new necessary and sufficient conditions for the multivariable outer factorization problem are formulated and proved.
\end{abstract}

\section{INTRODUCTION}

The Fejér-Riesz theorem for trigonometric polynomials $q(z)=\sum_{i=-n}^{n} q_{i} z^{i}$ over $\mathbb{C}$ states that $q(z) \geq 0, z \in \mathbb{T}$, if and only if there exists a polynomial $p(z)=\sum_{i=0}^{n} p_{i} z^{i}$ so that $q(z)=|p(z)|^{2}, z \in \mathbb{T}$. In addition, one may choose $p$ to be void of roots inside the open unit circle $\mathbb{D}$ (that is, $p$ is outer). Though simple to state and prove (use the fundamental theorem of algebra; see, for example, [15]), the lemma has many useful applications; for example, in filter design, $H^{\infty}$ control, and wavelet theory. The first generalizations of the lemma involved matrix-valued trigonometric polynomials (16], 12]) and subsequently operator-valued trigonometric polynomials (in [11] a compactness condition appears, in [17] the general operator case is done).

The present paper grew out of an interest in a multivariate analog of the FejérRiesz theorem. As is well known, extensions of such results to several variables are far from straightforward. One of the earliest efforts in this direction is Hilbert's wellknown observation that not all nonnegative polynomials in several real variables are necessarily sums of squares of polynomials. While Hilbert's result concerns polynomials on $\mathbb{R}^{d}$, a similar phenomenon occurs in the setting of trigonometric polynomials on the $d$-torus $\mathbb{T}^{d}$, where $\mathbb{T}=\{z \in \mathbb{C}:|z|=1\}$. Indeed, it follows from Hilbert's result (see [3] and [19]) that a trigonometric polynomial $q(z)$ in $d$ variables of degree $\left(n_{1}, \ldots, n_{d}\right)$ that takes on nonnegative values on $\mathbb{T}^{d}$, is not necessarily of the form

$$
q(z)=\sum_{i=1}^{k}\left|p_{i}(z)\right|^{2}, z \in \mathbb{T}^{d},
$$

Received by the editors March 1, 2004.

2000 Mathematics Subject Classification. Primary 47A68, 47B35, 15A48.

The first author's research was supported by the Engineering and Physical Sciences Research Council (EPSRC) and by the European Community's Human Potential Programme Under Contract HPRN-CT-2000-00116 (Analysis And Operators).

The second author's research was supported in part by the National Science Foundation (NSF), as well as a Faculty Research Assignment (FRA) Grant from the College of William \& Mary. 
where $p_{i}$ are polynomials of degree $\left(n_{1}, \ldots, n_{d}\right)$. It turns out (see [5]; see also [14, 13]) that checking whether $q$ can be factored in this way is a semidefinite feasibility problem. In this paper, we investigate which multivariable trigonometric polynomials are single squares; that is, we would like $k=1$ in the above representation (1.1) with similar restrictions on the degree of the polynomial in the factorization.

As might be expected, not putting any restrictions on the degrees of the polynomials $p_{i}$ in (1.1) enables more nonnegative trigonometric polynomials to be factored. In fact, in 8 it was shown that any strictly positive trigonometric polynomial (i.e., $q(z)>0, z \in \mathbb{T}^{d}$ ) allows a representation (1.1), where $p_{i}$ are polynomials of potentially very high degree. This in turn relates to factorization of real polynomials as sums of squares of rational functions with fixed denominators [8]. An important tool in [8] is the use of Schur complements. Inspired by this we also use the Schur complement as our main tool. This allows for a very simple proof of Rosenblum's operator-valued Fejér-Riesz theorem. The main observation in this proof is that the sequence of finitely supported Schur complements of a banded positive semidefinite Toeplitz operator has a very simple inheritance structure (see Proposition 3.1), a result that was not yet observed in [8]. In fact, beyond a certain matrix size (determined by the number of nonzero diagonals) as the Schur complement is increased one dimension in size, it is constructed by bordering the previous Schur complement with the coefficients of the underlying trigonometric polynomial. Recognizing this, the task becomes how to determine the multivariate analog of this inheritance structure. Clearly, there are now many canonical shifts; how does one use these? As we will see, for the multivariate trigonometric polynomial to have an outer factorization of the required type, the Schur complement of the corresponding Toeplitz operator needs to decompose in a certain way. Subsequently, to obtain the next Schur complement, the different terms in this decomposition need to be shifted in different ways. Bordering the result with the coefficients of the trigonometric polynomial then yields the next Schur complement. The precise statement is given in Theorem 4.3. In [10] a two-variable Fejér-Riesz result was obtained for the case when the trigonometric polynomial is strictly positive and scalar valued. The approach there was completely different, as the factorization result was obtained as a corollary of a result on bivariate autoregressive filters.

The paper is organized as follows. In Section 2 we derive several useful new properties of Schur complements. In Section 3 we use these newly-observed properties to provide easy proofs for Rosenblum's version of the operator-valued Fejér-Riesz theorem and the existence of inner-outer factorizations. In Section 4 the multivariate case is addressed.

\section{Auxiliary Results on Schur COMPlements}

Recall that if $\mathcal{H}_{1}$ and $\mathcal{H}_{2}$ are Hilbert spaces and

$$
M=\left(\begin{array}{cc}
A & B \\
B^{*} & C
\end{array}\right): \mathcal{H}_{1} \oplus \mathcal{H}_{2} \rightarrow \mathcal{H}_{1} \oplus \mathcal{H}_{2}
$$

is a positive semidefinite operator, then there exists a unique contraction $G$ : $\overline{\operatorname{ran}}(C) \rightarrow \overline{\operatorname{ran}}(A)$ such that $B=A^{1 / 2} G C^{1 / 2}$. The Schur complement $S$ of $M$ supported on $\mathcal{H}_{1}$ is defined to be the positive semidefinite operator $A^{1 / 2}\left(1-G G^{*}\right) A^{1 / 2}$. 
An alternative way to define the Schur complement of $M$ supported on $\mathcal{H}_{1}$ is via

$$
\langle S f, f\rangle=\inf \left\{\left\langle\left(\begin{array}{cc}
A & B \\
B^{*} & C
\end{array}\right)\left(\begin{array}{l}
f \\
g
\end{array}\right),\left(\begin{array}{l}
f \\
g
\end{array}\right)\right\rangle: g \in \mathcal{H}_{2}\right\} ;
$$

that is, it is the largest positive semidefinite operator which may be subtracted from $A$ in (2.1) such that the resulting operator matrix remains positive semidefinite.

A few words are required on our notation. We will typically number rows and columns of an $n \times n$ matrix with $0, \ldots, n-1$. For $\Lambda \subseteq\{0, \ldots, n-1\}$ and an $n \times n$ operator matrix $M$, we write $S(M ; \Lambda)$ (or $S(\Lambda)$ when there is no chance of confusion) for the Schur complement supported on rows and columns labeled by elements of $\Lambda$. It is usual to view $S(\Lambda)$ as an $m \times m$ matrix, where $m=\operatorname{card} \Lambda$, however it is often useful to take $S(\Lambda)$ to be an $n \times n$ matrix. If $\Lambda=\left\{n_{0}, \ldots, n_{m-1}\right\}$, then this is done by putting the $(j, k)$ entry of $S(\Lambda)$ as an $m \times m$ matrix into the $\left(n_{j}, n_{k}\right)$ place and padding with zeros. We use the same notation for both versions of the Schur complement, since it should be clear from the context which we are using. Finally, as a further bit of notational convenience, we write $S(M ; k)$ (or $S(k))$ when $\Lambda=\{0, \ldots, k\}$.

Lemma 2.1. Let $M: \mathcal{H}_{1} \oplus \mathcal{H}_{2} \rightarrow \mathcal{H}_{1} \oplus \mathcal{H}_{2}$ be factored as

$$
M=\left(\begin{array}{cc}
A & B \\
B^{*} & C
\end{array}\right)=\left(\begin{array}{cc}
P^{*} & Q^{*} \\
0 & R^{*}
\end{array}\right)\left(\begin{array}{cc}
P & 0 \\
Q & R
\end{array}\right),
$$

where $P: \mathcal{H}_{1} \rightarrow \mathcal{K}_{1}, Q: \mathcal{H}_{1} \rightarrow \mathcal{K}_{2}$, and $R: \mathcal{H}_{2} \rightarrow \mathcal{K}_{2}$. Then $S(0)$ equals $P^{*} P$ if and only if ran $Q \subseteq \overline{r a n} R$. Furthermore, for any $P$ such that $P^{*} P=S(0)$ and any $R$ such that $R^{*} R=C$, there is a $Q$ such that (2.2) holds.

Proof. Since $C=R^{*} R$, there is an isometry $V: \overline{\operatorname{ran}} R \rightarrow \overline{\operatorname{ran}} C^{1 / 2}$ such that $C^{1 / 2}=V R$. Clearly $\left(\begin{array}{cc}A & B \\ B^{*} & C\end{array}\right) \geq 0$, so there is a contraction $G: \overline{\operatorname{ran}} C^{1 / 2} \rightarrow \overline{\operatorname{ran}} A^{1 / 2}$ with $B=A^{1 / 2} G C^{1 / 2}$, and consequently $B=A^{1 / 2} G V R$. We also have $B=Q^{*} R$, so the assumption that $\operatorname{ran} Q \subseteq \overline{\operatorname{ran}} R$ implies that $A^{1 / 2} G V=Q^{*}$. Moreover, since $V V^{*}=1_{\overline{\mathrm{ran}}} C^{1 / 2}$, we get that $\bar{A}^{1 / 2} G=Q^{*} V^{*}$. We calculate the Schur complement

$$
\begin{aligned}
S(0) & =A^{1 / 2}\left(1-G G^{*}\right) A^{1 / 2}=A-Q^{*} V^{*} V Q=A-Q^{*} 1_{\overline{\text { ran }} R^{1 / 2}} Q \\
& =A-Q^{*} Q=P^{*} P+Q^{*} Q-Q^{*} Q=P^{*} P .
\end{aligned}
$$

Conversely assume that $P^{*} P=S(0)$. It follows from (2.2) that

$$
\left(\begin{array}{cc}
A & B \\
B^{*} & C
\end{array}\right)-\left(\begin{array}{cc}
P^{*} P & 0 \\
0 & 0
\end{array}\right)=\left(\begin{array}{cc}
Q^{*} Q & Q^{*} R \\
R^{*} Q & R^{*} R
\end{array}\right) .
$$

If we set $V_{R}, V_{Q}$ to be the inclusions of $\overline{\operatorname{ran}} R$ and $\overline{\operatorname{ran}} Q$ into $\mathcal{K}_{2}$, respectively, then $Q^{*} R=Q^{*} G R$, where $G=V_{Q}^{*} V_{R}$. By construction the Schur complement of the right side of (2.3) is zero, which implies that

$$
0=1_{\overline{\operatorname{ran}} Q}-G G^{*}=1_{\overline{\operatorname{ran}} Q}-V_{Q}^{*} V_{R} V_{R}^{*} V_{Q}=V_{Q}^{*}\left(1-P_{\overline{\operatorname{ran}} R}\right) V_{Q},
$$

where $P_{\overline{\operatorname{ran}} R}$ is the orthogonal projection onto $\overline{\operatorname{ran}} R$. Thus $P_{\overline{\operatorname{ran}} R} \mid \overline{\operatorname{ran}} Q=1_{\overline{\operatorname{ran}} Q}$, and hence $\operatorname{ran} Q \subseteq \operatorname{ran} P_{\overline{\text { ran }} R}=\overline{\operatorname{ran}} R$.

Finally, suppose $P^{*} P=S(0)$ and $R^{*} R=C$. Then $A-P^{*} P \geq 0$ and $B=$ $A^{1 / 2} G R$ for some contraction $G: \overline{\operatorname{ran}} R \rightarrow \overline{\operatorname{ran}} A^{1 / 2}$. Hence

$$
P^{*} P=A^{1 / 2}\left(1-G G^{*}\right) A^{1 / 2}
$$


and there exists $D_{G}$ such that $D_{G} D_{G}^{*}=1-G G^{*}$ and $P=A^{1 / 2} D_{G}$. Then setting $Q^{*}=A^{1 / 2} G$, we have $M=\left(\begin{array}{cc}P^{*} & Q^{*} \\ 0 & R^{*}\end{array}\right)\left(\begin{array}{ll}P & 0 \\ Q & R\end{array}\right)$, and $\operatorname{ran} Q \subseteq \operatorname{ran} G^{*} \subseteq \overline{\operatorname{ran}} R$.

Lemma 2.2. Suppose

$$
M=\left(\begin{array}{ccc}
A & B & C \\
B^{*} & D & E \\
C^{*} & E^{*} & F
\end{array}\right)=\left(\begin{array}{ccc}
P^{*} & Q^{*} & R^{*} \\
0 & S^{*} & T^{*} \\
0 & 0 & U^{*}
\end{array}\right)\left(\begin{array}{ccc}
P & 0 & 0 \\
Q & S & 0 \\
R & T & U
\end{array}\right),
$$

where $M$ is acting on $\mathcal{H}_{1} \oplus \mathcal{H}_{2} \oplus \mathcal{H}_{3}$. Then

$$
S(1)-S(0)=\left(\begin{array}{c}
Q^{*} \\
S^{*}
\end{array}\right)\left(\begin{array}{ll}
Q & S
\end{array}\right)
$$

if and only if

$$
\operatorname{ran} Q \subseteq \overline{\operatorname{ran}} S \quad \text { and } \quad \operatorname{ran} T \subseteq \overline{\operatorname{ran}} U .
$$

Furthermore there exists a factorization of $M$ as in (2.4) with the factors operators on $\mathcal{H}_{1} \oplus \mathcal{H}_{2} \oplus \mathcal{H}_{3}$ such that (2.5) and (2.6) hold, $\left(\begin{array}{cc}P^{*} & Q^{*} \\ 0 & S^{*}\end{array}\right)\left(\begin{array}{cc}P & 0 \\ Q & S\end{array}\right)=S(1)$ and $P^{*} P=S(0)=S(S(1) ; 0)$.

Proof. To begin with, suppose (2.5) holds. Then if $\tilde{P}^{*} \tilde{P}=S(0)$, we have

$$
S(1)=\left(\begin{array}{cc}
\tilde{P}^{*} & Q^{*} \\
0 & S^{*}
\end{array}\right)\left(\begin{array}{cc}
\tilde{P} & 0 \\
Q & S
\end{array}\right) \text {. }
$$

As $U^{*} U=F$, by Lemma 2.1 there exist $\tilde{R}$ and $\tilde{T}$ such that

$$
M=\left(\begin{array}{ccc}
\tilde{P}^{*} & Q^{*} & \tilde{R}^{*} \\
0 & S^{*} & \tilde{T}^{*} \\
0 & 0 & U^{*}
\end{array}\right)\left(\begin{array}{ccc}
\tilde{P} & 0 & 0 \\
Q & S & 0 \\
\tilde{R} & \tilde{T} & U
\end{array}\right) .
$$

By Lemma 2.1

$$
\operatorname{ran}\left(\begin{array}{c}
Q \\
\tilde{R}
\end{array}\right) \subseteq \overline{\operatorname{ran}}\left(\begin{array}{cc}
S & 0 \\
\tilde{T} & U
\end{array}\right)
$$

and

$$
\operatorname{ran}\left(\begin{array}{cc}
\tilde{R} & \tilde{T}
\end{array}\right) \subseteq \overline{\operatorname{ran}} U
$$

Hence $\operatorname{ran} \tilde{T} \subseteq \overline{\operatorname{ran}} U$ and so

$$
\overline{\operatorname{ran}}\left(\begin{array}{cc}
S & 0 \\
\tilde{T} & U
\end{array}\right)=\overline{\operatorname{ran}} S \oplus \overline{\operatorname{ran}} U .
$$

Thus $\operatorname{ran} Q \subseteq \overline{\operatorname{ran}} S$.

Next observe that $D=S^{*} S+T^{*} T=S^{*} S+\tilde{T}^{*} \tilde{T}$ and so there is an isometry $V_{T}: \overline{\operatorname{ran}} T \rightarrow \overline{\operatorname{ran}} \tilde{T}$ such that $T^{*}=\tilde{T}^{*} V_{T}^{*}$. Also $\operatorname{ran} \tilde{T} \subseteq \overline{\operatorname{ran}} U$ implies that $\operatorname{ran} V_{T} \subseteq \overline{\operatorname{ran}} U$. Thus $V_{T}$ is an isometry from $\overline{\operatorname{ran}} T$ into $\overline{\operatorname{ran}} U$. But $U^{*} T=E^{*}=$ $U^{*} \tilde{T}=U^{*} V_{T} T$, so $V_{T}=1_{\overline{\operatorname{ran}} T}$ and $\operatorname{ran} T \subseteq \overline{\operatorname{ran}} U$.

Now conversely assume we have a factorization of $M$ as in (2.4), where (2.6) holds. Set

$$
L=\left(\begin{array}{cc}
D & E \\
E^{*} & F
\end{array}\right)=\left(\begin{array}{cc}
S^{*} & T^{*} \\
0 & U^{*}
\end{array}\right)\left(\begin{array}{cc}
S & 0 \\
T & U
\end{array}\right)
$$


Using Lemma 2.1 suppose $\tilde{G}$ is any other operator matrix satisfying $\tilde{G}^{*} \tilde{G}=M$ with

$$
\tilde{G}=\left(\begin{array}{ccc}
\tilde{P} & 0 & 0 \\
\tilde{Q} & \tilde{S} & 0 \\
\tilde{R} & \tilde{T} & U
\end{array}\right),
$$

where

$$
S(1)=\left(\begin{array}{cc}
\tilde{P}^{*} & \tilde{Q}^{*} \\
0 & \tilde{S}^{*}
\end{array}\right)\left(\begin{array}{cc}
\tilde{P} & 0 \\
\tilde{Q} & \tilde{S}
\end{array}\right)
$$

and $\tilde{P}$ chosen so that $S(S(1) ; 0)=\tilde{P}^{*} \tilde{P}$. Note that

$$
L=\left(\begin{array}{cc}
\tilde{S}^{*} & \tilde{T}^{*} \\
0 & U^{*}
\end{array}\right)\left(\begin{array}{cc}
\tilde{S} & 0 \\
\tilde{T} & U
\end{array}\right) .
$$

Since by assumption $\operatorname{ran} T \subseteq \overline{\operatorname{ran}} U$, we have $S^{*} S=S(L ;\{1\}) \geq \tilde{S}^{*} \tilde{S}$. On the other hand, since

$$
S(1) \geq\left(\begin{array}{cc}
P^{*} & Q^{*} \\
0 & S^{*}
\end{array}\right)\left(\begin{array}{ll}
P & 0 \\
Q & S
\end{array}\right),
$$

we also have $\tilde{S}^{*} \tilde{S} \geq S^{*} S$. Hence $\tilde{S}^{*} \tilde{S}=S^{*} S$. Thus $V S=\tilde{S}$ for some isometry $V: \overline{\operatorname{ran}} S \rightarrow \overline{\operatorname{ran}} \overline{\tilde{S}}$. Since we have chosen $S(S(1) ; 0)=\tilde{P}^{*} \tilde{P}$, by Lemma [2.1] $\operatorname{ran} \tilde{Q} \subseteq \overline{\operatorname{ran}} \tilde{S}$. Moreover,

$$
0 \leq\left(\begin{array}{cc}
\tilde{P}^{*} \tilde{P}+\tilde{Q}^{*} \tilde{Q} & \tilde{Q}^{*} S \\
S^{*} \tilde{Q} & S^{*} S
\end{array}\right)-\left(\begin{array}{cc}
P^{*} P+Q^{*} Q & Q^{*} S \\
S^{*} Q & S^{*} S
\end{array}\right)
$$

and $\tilde{S}^{*} \tilde{S} \geq S^{*} S$ imply that $0=\tilde{Q}^{*} \tilde{S}-Q^{*} S=\left(\tilde{Q}^{*} V-Q^{*}\right) S$. As $\operatorname{ran} Q \subseteq \overline{\operatorname{ran}} S$ it follows that $\tilde{Q}^{*} V=Q^{*}$. Thus, in particular, $\tilde{Q}^{*} \tilde{Q}=Q^{*} Q$. But then we obtain that

$$
\tilde{P}^{*} \tilde{P} \geq P^{*} P
$$

Observe that (2.8) will be true no matter what the original factorization of $M$ in (2.4) is as long as the range conditions in (2.6) are satisfied.

Now instead consider the factorization $M=G^{*} G^{\prime}$, where

$$
G^{\prime}=\left(\begin{array}{ccc}
P^{\prime} & 0 & 0 \\
Q^{\prime} & S & 0 \\
R^{\prime} & T & U
\end{array}\right)
$$

with $P^{\prime *} P^{\prime}=S(0)$. Such a factorization is possible by Lemma 2.1 Since by assumption $\operatorname{ran} T \subseteq \overline{\operatorname{ran}} U$, we have

$$
\overline{\operatorname{ran}}\left(\begin{array}{cc}
S & 0 \\
T & U
\end{array}\right) \subseteq \overline{\operatorname{ran}} S \oplus \overline{\operatorname{ran}} U
$$

Also by Lemma 2.1 then,

$$
\operatorname{ran}\left(\begin{array}{l}
Q^{\prime} \\
R^{\prime}
\end{array}\right) \subseteq \overline{\operatorname{ran}} S \oplus \overline{\operatorname{ran}} U
$$

and hence $\operatorname{ran} Q^{\prime} \subseteq \overline{\operatorname{ran}} S$. So the conditions in (2.6) are satisfied for this factorization, and hence as noted above, we must have $\tilde{P}^{*} \tilde{P} \geq P^{\prime *} P^{\prime}$. But by definition of the Schur complement, $P^{\prime *} P^{\prime} \geq \tilde{P}^{*} \tilde{P}$, so we have equality. Consequently, (2.5) holds. 
Finally, using Lemma 2.1 there is a factorization

$$
L=\left(\begin{array}{cc}
S^{*} & T^{*} \\
0 & U^{*}
\end{array}\right)\left(\begin{array}{cc}
S & 0 \\
T & U
\end{array}\right)
$$

where $\operatorname{ran} T \subseteq \overline{\operatorname{ran}} U \subseteq \mathcal{H}_{3}$, so that $\overline{\operatorname{ran}}\left(\begin{array}{cc}S & T \\ 0 & U\end{array}\right)=\overline{\operatorname{ran}} S \oplus \overline{\operatorname{ran}} U \subseteq \mathcal{H}_{2} \oplus \mathcal{H}_{3}$. Again by Lemma 2.1 there exists $P: \mathcal{H}_{1} \rightarrow \mathcal{H}_{1}$ such that $P^{*} P=S(0)$ and (2.4) holds. Consequently $\operatorname{ran}\left(\begin{array}{l}Q \\ R\end{array}\right) \subseteq \overline{\operatorname{ran}} S \oplus \overline{\operatorname{ran}} U$, giving $\operatorname{ran} Q \subseteq \overline{\operatorname{ran}} S$.

It is now clear that the factorization in 2.4 with these choices of $P, Q, R, S, T$ and $U$ satisfies the last statement of the theorem.

Corollary 2.3. Let $M \geq 0$ be an $n \times n$ operator matrix, $J \subseteq K \subseteq\{0 \ldots n-1\}$. Then

$$
S(J)=S(S(K) ; J) .
$$

Proof. Let $I_{1}=J, I_{2}=K \backslash J$ and $I_{3}=\{0, \ldots, n-1\} \backslash K$. Writing $M$ as a $3 \times 3$ block matrix with respect to the partition

$$
\{0, \ldots, n-1\}=I_{1} \cup I_{2} \cup I_{3},
$$

the corollary follows directly from Lemma 2.2 ,

Corollary 2.4. Given $M \geq 0$ an $n \times n$ operator matrix, there is a factorization $M=P^{*} P$, where

$$
P=\left(\begin{array}{ccccc}
P_{00} & 0 & \cdots & \cdots & 0 \\
P_{10} & P_{11} & 0 & \cdots & 0 \\
\vdots & \vdots & \ddots & \ddots & \vdots \\
\vdots & \vdots & \ddots & \ddots & 0 \\
P_{n-1,0} & P_{n-1,1} & \cdots & \cdots & P_{n-1, n-1}
\end{array}\right)
$$

where $\overline{\operatorname{ran}} P=\overline{\operatorname{ran}} P_{00} \oplus \cdots \oplus \overline{\operatorname{ran}} P_{n-1, n-1}$ and such that if $P_{k}$ is the truncation of $P$ to the upper left $(k+1) \times(k+1)$ corner, then $S(k)=P_{k}^{*} P_{k}, k=0, \ldots, n$.

The above result also appears in [6].

Lemma 2.5. Let

$$
\left(\begin{array}{cc}
P^{*} & Q^{*} \\
0 & R^{*}
\end{array}\right)\left(\begin{array}{cc}
P & 0 \\
Q & R
\end{array}\right)=\left(\begin{array}{cc}
\tilde{P}^{*} & \tilde{Q}^{*} \\
0 & \tilde{R}^{*}
\end{array}\right)\left(\begin{array}{cc}
\tilde{P} & 0 \\
\tilde{Q} & \tilde{R}
\end{array}\right),
$$

and suppose that $\operatorname{ran} Q \subseteq \overline{\operatorname{ran}} R$. Then there is a unique isometry

$$
\left(\begin{array}{cc}
V_{11} & 0 \\
V_{21} & V_{22}
\end{array}\right)
$$

acting on $\overline{\operatorname{ran}} P \oplus \overline{\operatorname{ran}} R$ so that

$$
\left(\begin{array}{cc}
\tilde{P} & 0 \\
\tilde{Q} & \tilde{R}
\end{array}\right)=\left(\begin{array}{cc}
V_{11} & 0 \\
V_{21} & V_{22}
\end{array}\right)\left(\begin{array}{ll}
P & 0 \\
Q & R
\end{array}\right) .
$$

Proof. It is a standard result that $A^{*} A=B^{*} B$ if and only there exist an isometry $V: \overline{\operatorname{ran}} B \rightarrow \overline{\operatorname{ran}} A$ so that $V B=A$. The operator $V$ is uniquely determined by 
setting $V(B x)=A x$ for every $B x \in \operatorname{ran} B$, and extending $V$ to $\overline{\operatorname{ran}} B$ by continuity. Thus (2.10) implies the existence of an isometry $V=\left(V_{i j}\right)_{i, j=1}^{2}$ satisfying

$$
\left(\begin{array}{ll}
\tilde{P} & 0 \\
\tilde{Q} & \tilde{R}
\end{array}\right)=\left(\begin{array}{ll}
V_{11} & V_{12} \\
V_{21} & V_{22}
\end{array}\right)\left(\begin{array}{ll}
P & 0 \\
Q & R
\end{array}\right) .
$$

It remains to show that $V_{12}=0$. Note that (2.12) implies that $V_{22} R=\tilde{R}$. Combining this with (2.10) we get that

$$
R^{*} R=\tilde{R}^{*} \tilde{R}=R^{*} V_{22}^{*} V_{22} R
$$

and thus

$$
R^{*}\left(I_{\overline{\mathrm{ran}} R}-V_{22}^{*} V_{22}\right) R=0 .
$$

As $\operatorname{ran} Q \subseteq \overline{\operatorname{ran}} R$ we have that

$$
\overline{\operatorname{ran}}\left(\begin{array}{ll}
P & 0 \\
Q & R
\end{array}\right)=\overline{\operatorname{ran}} P \oplus \overline{\operatorname{ran}} R .
$$

Thus $V_{22}$ and $V_{12}$ act on $\overline{\text { ran }} R$. From (2.13) we now obtain that $V_{22}$ is an isometry on $\overline{\operatorname{ran}} R$. But then, since $V$ is an isometry, we must have that $V_{12}=0$.

In the following lemma we consider a positive semidefinite operator on $\mathcal{H}_{1} \oplus$ $\mathcal{H}_{2} \oplus \mathcal{H}_{3} \oplus \mathcal{H}_{4}$, the $\mathcal{H}_{k}$ 's Hilbert spaces.

Lemma 2.6. Let $A=\left(A_{i j}\right)_{i, j=1}^{4} \geq 0$. Then we have that

$$
[S(2)]_{21}=0
$$

if and only if

$$
S(2)=S(1)+S(\{0,2\})-S(0) .
$$

Proof. The direction (2.15) $\Rightarrow 2.14$ is trivial.

By Corollary 2.4, there is a lower triangular $3 \times 3$ operator matrix

$$
P=\left(\begin{array}{ccc}
P_{00} & 0 & 0 \\
P_{10} & P_{11} & 0 \\
P_{20} & P_{21} & P_{22}
\end{array}\right)
$$

such that $S(2)=P^{*} P, S(1)=\left(\begin{array}{cc}P_{00}^{*} & P_{10}^{*} \\ 0 & P_{11}^{*}\end{array}\right)\left(\begin{array}{cc}P_{00} & 0 \\ P_{10} & P_{11}\end{array}\right)$, and $S(0)=P_{00}^{*} P_{00}$. Also, $\operatorname{ran} P_{10} \subseteq \overline{\operatorname{ran}} P_{11}$ and $\operatorname{ran} P_{20}, \operatorname{ran} P_{21} \subseteq \overline{\operatorname{ran}} P_{22}$. Thus $[S(2)]_{21}=0$ is equivalent to $P_{21}=0$. Interchanging the order of rows 1 and 2 and columns 1 and 2 , we have

$$
S(\{0,2,1\})=\left(\begin{array}{ccc}
P_{00}^{*} & P_{20}^{*} & P_{10}^{*} \\
0 & P_{22}^{*} & 0 \\
0 & 0 & P_{11}^{*}
\end{array}\right)\left(\begin{array}{ccc}
P_{00} & 0 & 0 \\
P_{20} & P_{22} & 0 \\
P_{10} & 0 & P_{11}
\end{array}\right) .
$$

Since ran $\left(\begin{array}{ll}P_{10} & 0\end{array}\right) \subseteq \overline{\text { ran }} P_{11}$, by Lemma 2.1

$$
S(\{0,2\})=\left(\begin{array}{cc}
P_{00}^{*} & P_{20}^{*} \\
0 & P_{22}^{*}
\end{array}\right)\left(\begin{array}{cc}
P_{00} & 0 \\
P_{20} & P_{22}
\end{array}\right) .
$$

A direct calculation verifies the equality in 2.15).

By relabeling and grouping as we did in the proof of Corollary 2.3, we obtain the following. 
Corollary 2.7. Suppose $M \geq 0$ is an $n \times n$ operator matrix, $K \cup J=N \subseteq$ $\{0, \ldots n-1\}$. Then

$$
S(N)=S(K)+S(J)-S(K \cap J)
$$

if and only if

$$
\begin{gathered}
{[S(N)]_{k, j}=0} \\
(k, j) \in(N \times N) \backslash((K \times K) \cup(J \times J)) \\
=[(K \backslash(K \cap J)) \times(J \backslash(K \cap J))] \cup[(J \backslash(K \cap J) \times(K \backslash(K \cap J))] .
\end{gathered}
$$

\section{ONE VARIABLE OUTER AND INNER-OUTER FACTORIZATION}

In this section we will provide new proofs for several one-variable factorization results. These proofs are based on the properties of Schur complements.

Given a Hilbert space $\mathcal{H}$ let $H_{\mathcal{H}}^{2}(\mathbb{D})$ denote the Hardy space of $\mathcal{H}$-valued functions analytic in the unit disk with square integrable boundary values. These functions will be identified with their boundary values whenever convenient. Given a pair of Hilbert spaces $\mathcal{H}, \mathcal{K}$, let $\mathbf{L}(\mathcal{H}, K)$ stand for the Banach space of bounded operators acting $\mathcal{H} \rightarrow \mathcal{K}$. We will write $\mathbf{L}(\mathcal{H})$ instead of $\mathbf{L}(\mathcal{H}, \mathcal{H})$. As usual, $H_{\mathbf{L}(\mathcal{H}, \mathcal{K})}^{\infty}(\mathbb{D})$ stands for the set of all bounded analytic $\mathbf{L}(\mathcal{H}, K)$-valued functions on $\mathbb{D}$. With the operator-valued function $F \in H_{\mathbf{L}(\mathcal{H}, \mathcal{K})}^{\infty}(\mathbb{D})$, we associate the operator $M_{F}$ : $H_{\mathcal{H}}^{2}(\mathbb{D}) \rightarrow H_{\mathcal{K}}^{2}(\mathbb{D})$ of multiplication by $F$; that is, $M_{F} g(z)=F(z) g(z)$. The function $F$ is called outer if the corresponding multiplication operator $M_{F}$ has dense range in $H_{\mathcal{M}}^{2}(\mathbb{D})$ for some subspace $\mathcal{M}$ of $\mathcal{K}$, and this reduces to the usual definition when $\mathcal{H}$ and $\mathcal{K}$ are $\mathbb{C}$. For $Q \in L_{\mathbf{L}(\mathcal{H})}^{\infty}(\mathbb{T})$, we consider the Toeplitz operator $T_{Q}: H_{\mathcal{H}}^{2}(\mathbb{D}) \rightarrow H_{\mathcal{H}}^{2}(\mathbb{D})$ defined via $T_{Q} f=\Pi_{+}(Q f)$, where $\Pi_{+}$the projection is from $L_{\mathcal{H}}^{2}(\mathbb{T})$ onto $H_{\mathcal{H}}^{2}(\mathbb{D})$. We shall often represent $T_{Q}$ via the Toeplitz operator matrix

$$
T_{Q} \equiv\left(\begin{array}{ccc}
Q_{0} & Q_{-1} & \cdots \\
Q_{1} & \ddots & \ddots \\
\vdots & \ddots &
\end{array}\right),
$$

where we make the obvious identification of $f(z)=\sum_{0}^{\infty} f_{k} z^{k} \in H_{\mathcal{H}}^{2}(\mathbb{D})$ with $\operatorname{col}\left(f_{k}\right)_{0}^{\infty} \in \ell_{\mathcal{H}}^{2}\left(\mathbb{N}_{0}\right)$, where $f_{j} \in \mathcal{H}$ and $\|f\|:=\sqrt{\sum_{j=0}^{\infty}\left\|f_{j}\right\|^{2}}<\infty$. We view the matrix as an operator matrix with rows and columns indexed by $\mathbb{N}_{0}=\{0,1, \ldots\}$. In addition, we shall often use the identification

$$
T_{Q}=\left(\begin{array}{cc}
Q_{0} & \operatorname{row}\left(Q_{-i}\right)_{i \geq 1} \\
\operatorname{col}\left(Q_{i}\right)_{i \geq 1} & T_{Q}
\end{array}\right) .
$$

In other words, the operator $L: z H_{\mathcal{H}}^{2}(\mathbb{D}) \rightarrow z H_{\mathcal{H}}^{2}(\mathbb{D})$ defined by $(L f)(z)=$ $z \Pi_{+}\left(Q z^{-1} f\right)$ will at times be identified with $T_{Q}$. Following the notation from the previous section, for $\Lambda \subset \mathbb{N}_{0}$ we let $S\left(T_{Q} ; \Lambda\right.$ ) (or $S(\Lambda)$ when no confusion is possible) denote the Schur complement of $T_{Q}$ supported on rows and columns indexed by $\Lambda$. In addition, $S(k)$ is shorthand for $S(\{0, \ldots, k\})$.

We first address the effect that the Toeplitz structure has on the Schur complements. 
Proposition 3.1. Consider the positive semidefinite Toeplitz operator

$$
T_{Q}=\left(Q_{i-j}\right)_{i, j=0}^{\infty}
$$

acting on $\ell_{\mathcal{H}}^{2}\left(\mathbb{N}_{0}\right)$. Then the Schur complements $S(m)$ of $T_{Q}$ satisfy the recurrence relation

$$
S(m)=\left(\begin{array}{cc}
A & B^{*} \\
B & S(m-1)
\end{array}\right),
$$

for an appropriate choice of $A: \mathcal{H} \rightarrow \mathcal{H}$ and $B: \mathcal{H} \rightarrow \mathcal{H}^{m}$. When $Q_{j}=0, j \geq m+1$, then $A=Q_{0}$ and $B=\operatorname{col}\left(Q_{i}\right)_{i=1}^{m}$.

Proof. By the definition of Schur complement

$$
T_{Q}-\left(\begin{array}{cc}
S(m) & 0 \\
0 & 0
\end{array}\right) \geq 0 .
$$

Let us write

$$
S(m)=\left(\begin{array}{cc}
A & B^{*} \\
B & C
\end{array}\right): \mathcal{H} \oplus \mathcal{H}^{m} \rightarrow \mathcal{H} \oplus \mathcal{H}^{m}
$$

Leaving out row and column 0 in (3.4) yields

$$
T_{Q}-\left(\begin{array}{cc}
C & 0 \\
0 & 0
\end{array}\right) \geq 0
$$

where we used identification (3.2). This shows that $C \leq S(m-1)$. On the other hand, leaving out row and columns $1, \ldots, m$ in (3.4) yields

$$
\left(\begin{array}{cc}
Q_{0}-A & \operatorname{row}\left(Q_{j}^{*}\right)_{j \geq m+1} \\
\operatorname{col}\left(Q_{j}\right)_{j \geq m+1} & T_{Q}
\end{array}\right) \geq 0
$$

Hence

$$
A \leq S\left(\left(\begin{array}{cc}
Q_{0} & \operatorname{row}\left(Q_{j}^{*}\right)_{j \geq m+1} \\
\operatorname{col}\left(Q_{j}\right)_{j \geq m+1} & T_{Q}
\end{array}\right) ; 0\right)=: \tilde{A} .
$$

Note that when $Q_{j}=0, j \geq m+1$, we have that $\tilde{A}=Q_{0}$. Now consider the operator matrix

$$
\left(\begin{array}{ccc}
Q_{0}-\tilde{A} & X & \operatorname{row}\left(Q_{j}^{*}\right)_{j \geq m+1} \\
X^{*} & \left(Q_{i-j}\right)_{i, j=1}^{m}-S(m-1) & \left(Q_{i-j}\right)_{i=1, j=m+1}^{m+1, \infty} \\
\operatorname{col}\left(Q_{j}\right)_{j \geq m+1} & \left(Q_{i-j}\right)_{i=m+1, j=1}^{\infty, m} & T_{Q}
\end{array}\right) .
$$

The existence of an operator $X$ making this into a positive semidefinite matrix is a variant of a standard operator matrix completion problem, and by [1] (see also, e.g., Theorem XVI.3.1 in [9] or [2]), there is always such an $X$. Note that when $\tilde{A}=Q_{0}$ we have necessarily that $X=0$. As (3.5) is positive semidefinite we obtain that

$$
\left(\begin{array}{cc}
\tilde{A} & \operatorname{row}\left(Q_{j}^{*}\right)_{j=1}^{m}-X \\
\operatorname{col}\left(Q_{j}\right)_{j=1}^{m}-X^{*} & S(m-1)
\end{array}\right) \leq S(m)=\left(\begin{array}{cc}
A & B^{*} \\
B & C
\end{array}\right) .
$$

This implies that $\tilde{A} \leq A$ and $S(m-1) \leq C$. As we also had that $A \leq \tilde{A}$ and $C \leq S(m-1)$, the equalities $A=\tilde{A}$ and $C=S(m-1)$ follow. This yields (3.3). Moreover, when $Q_{j}=0$ for $j \geq m+1$, we have that $\tilde{A}=Q_{0}$ and $X=0$, and thus $B=\operatorname{col}\left(Q_{i}\right)_{i=1}^{m}$. 
Remark. Note that the proof shows that the operator $A$ in (3.3) is given by

$$
A=S\left(\left(\begin{array}{cc}
Q_{0} & \operatorname{row}\left(Q_{j}^{*}\right)_{j \geq m+1} \\
\operatorname{col}\left(Q_{j}\right)_{j \geq m+1} & T_{Q}
\end{array}\right) ; 0\right) .
$$

Because of the inheritance principle observed in Proposition 3.1, the Schur complements of a Toeplitz operator allow a stationary $U L$ Cholesky decomposition.

Corollary 3.2. Consider the positive semidefinite Toeplitz operator

$$
T_{Q}=\left(Q_{i-j}\right)_{i, j=0}^{\infty}
$$

acting on $\ell_{\mathcal{H}}^{2}\left(\mathbb{N}_{0}\right)$. Then there exist operators $F_{0}, F_{1}, \ldots$ with $F_{i}: \mathcal{H} \rightarrow \overline{r a n} F_{0} \subseteq \mathcal{H}$ so that the Schur complements $S(m)$ of $T_{Q}$ satisfy

$$
S(m)=\left(\begin{array}{ccc}
F_{0}^{*} & \cdots & F_{m}^{*} \\
& \ddots & \vdots \\
& & F_{0}^{*}
\end{array}\right)\left(\begin{array}{ccc}
F_{0} & & \\
\vdots & \ddots & \\
F_{m} & \cdots & F_{0}
\end{array}\right), \quad m \geq 0 .
$$

Proof. We prove this by induction. When $m=0$ we may for instance choose $F_{0}=$ $(S(0))^{1 / 2}$. It follows from Proposition 3.1 that $(S(m))_{m, m}=(S(m-1))_{m-1, m-1}=$ $F_{0}^{*} F_{0}$, where in the last step we used the induction hypothesis. By Corollary 2.3 we have that $S(m-1)=S(S(m) ; m-1)$, and thus by Lemma 2.1 with

$$
P=\left(\begin{array}{ccc}
F_{0} & & \\
\vdots & \ddots & \\
F_{m-1} & \cdots & F_{0}
\end{array}\right), \quad R=F_{0}
$$

there exist $\left(\begin{array}{lll}G_{m} & \cdots & G_{1}\end{array}\right)$ so that

$$
S(m)=\left(\begin{array}{cccc}
F_{0}^{*} & \cdots & F_{m-1}^{*} & G_{m}^{*} \\
& \ddots & \vdots & \vdots \\
& & F_{0}^{*} & G_{1}^{*} \\
& & & F_{0}^{*}
\end{array}\right)\left(\begin{array}{ccccc}
F_{0} & & & \\
\vdots & \ddots & & \\
F_{m-1} & \cdots & F_{0} & \\
G_{m} & \cdots & G_{1} & F_{0}
\end{array}\right),
$$

and $\operatorname{ran}\left(G_{m} \quad \cdots \quad G_{1}\right) \subseteq \overline{\operatorname{ran}} F_{0}$. Comparing (3.7) with (3.3) along with the induction hypothesis yields

$$
\begin{aligned}
&\left(\begin{array}{cccc}
F_{0}^{*} & \cdots & F_{m-2}^{*} & G_{m-1}^{*} \\
& \ddots & \vdots & \vdots \\
& & F_{0}^{*} & G_{1}^{*} \\
& & F_{0}^{*}
\end{array}\right)\left(\begin{array}{cccc}
F_{0} & & & \\
\vdots & \ddots & & \\
F_{m-2} & \cdots & F_{0} & \\
G_{m-1} & \cdots & G_{1} & F_{0}
\end{array}\right)=S(m-1) \\
&=\left(\begin{array}{cccc}
F_{0}^{*} & \cdots & F_{m-2}^{*} & F_{m-1}^{*} \\
& \ddots & \vdots & \vdots \\
& & F_{0}^{*} & F_{1}^{*} \\
& & & F_{0}^{*}
\end{array}\right)\left(\begin{array}{ccccc}
F_{0} & & \\
\vdots & \ddots & & \\
F_{m-2} & \cdots & F_{0} & \\
F_{m-1} & \cdots & F_{1} & F_{0}
\end{array}\right),
\end{aligned}
$$

and thus

$$
F_{0}^{*}\left(\begin{array}{lll}
G_{m-1} & \cdots & G_{1}
\end{array}\right)=F_{0}^{*}\left(\begin{array}{lll}
F_{m-1} & \cdots & F_{1}
\end{array}\right) .
$$

As ran $\left(\begin{array}{lll}G_{m-1} & \cdots & G_{1}\end{array}\right) \subseteq \overline{\operatorname{ran}} F_{0}$ and $\operatorname{ran}\left(\begin{array}{lll}F_{m-1} & \cdots & F_{1}\end{array}\right) \subseteq \overline{\operatorname{ran}} F_{0}$, it follows that $G_{j}=F_{j}, j=1, \ldots, m-1$. By setting $F_{m}:=G_{m}$, we obtain the result. 
Before we come to our main items, let us develop some equivalent statements for outerness that follow directly from the Schur complement results. Analogously to (B.2), we shall use the identification

$$
T_{F}=\left(\begin{array}{cc}
F_{0} & 0 \\
\operatorname{col}\left(F_{j}\right)_{j \geq 1} & T_{F}
\end{array}\right) .
$$

Theorem 3.3. Let $F \in H_{\mathbf{L}(\mathcal{H}, \mathcal{K})}^{\infty}(\mathbb{D})$. Denote the Taylor coefficients of $F$ by $F_{j}$, $j \geq 0$. The following are equivalent:

(i) $F$ is outer;

(ii) $\overline{\operatorname{ran}} M_{F}=H_{\overline{\operatorname{ran}} F_{0}}^{2}(\mathbb{D})$;

(iii) $\operatorname{ran} \operatorname{col}\left(F_{j}\right)_{j \geq 1} \subset \overline{\operatorname{ran}} T_{F}$;

(iv) $S\left(T_{F}^{*} T_{F} ; 0\right)=F_{0}^{*} F_{0}$;

(v) for some $k \in \mathbb{N}_{0}$ we have that

$$
S\left(T_{F}^{*} T_{F} ; k\right)=\left(\begin{array}{ccc}
F_{0}^{*} & \cdots & F_{k}^{*} \\
& \ddots & \vdots \\
& & F_{0}^{*}
\end{array}\right)\left(\begin{array}{ccc}
F_{0} & & \\
\vdots & \ddots & \\
F_{k} & \cdots & F_{0}
\end{array}\right)
$$

(vi) for all $k \in \mathbb{N}_{0}$ equality (3.9) holds.

Proof. Clearly (ii) implies (i). For the implication (i) $\Rightarrow$ (ii), observe that if $\overline{\operatorname{ran}} M_{F}=H_{\mathcal{M}}^{2}(\mathbb{D})$, then $P_{0}\left(\overline{\operatorname{ran}} T_{F}\right)=\mathcal{M}$, where $P_{0}$ is the projection $F \mapsto F_{0}$. But when $h \in H_{\mathcal{M}}^{2}(\mathbb{D})$ we have that $P_{0}(F h)=F_{0} h(0) \in \operatorname{ran} F_{0}$. Moreover, since we may let $h(0)$ range over all elements in $\mathcal{H}$, we obtain that $\mathcal{M}=\overline{\operatorname{ran}} F_{0}$.

For (ii) $\Rightarrow$ (iii), note that given (ii) we get that

$$
\operatorname{ran} \operatorname{col}\left(F_{j}\right)_{j \geq 1} \subset\left(I-P_{0}\right) \ell_{\text {ran } F_{0}}^{2}\left(\mathbb{N}_{0}\right) \cong H_{\text {ran } F_{0}}^{2}(\mathbb{D})=\overline{\operatorname{ran}} T_{F} .
$$

Here we used the identification of a multiplication operator and its corresponding Toeplitz operator.

Next consider (iii) $\Rightarrow$ (ii). If $h_{0} \in \mathcal{H}$ we have that $\operatorname{col}\left(F_{j} h_{0}\right)_{j \geq 1} \in \overline{\operatorname{ran}} T_{F}$. Thus there exist $g_{i}$ so that $\lim _{i \rightarrow \infty} T_{F} g_{i}=\operatorname{col}\left(F_{j} h_{0}\right)_{j \geq 1}$. But then

$$
\left(\begin{array}{cc}
F_{0} & 0 \\
\operatorname{col}\left(F_{j}\right)_{j \geq 1} & T_{F}
\end{array}\right)\left(\begin{array}{c}
h_{0} \\
-g_{i}
\end{array}\right) \rightarrow\left(\begin{array}{c}
F_{0} h_{0} \\
0
\end{array}\right)
$$

Thus $F_{0} h_{0} \in \overline{\operatorname{ran}} M_{F}$. As $\overline{\text { ran }} M_{F}$ is closed under multiplication with $z$, we get that $H_{\overline{\text { ran }} F_{0}}^{2}(\mathbb{D}) \subseteq \overline{\operatorname{ran}} M_{F}$. The inclusion $\overline{\operatorname{ran}} M_{F} \subseteq H_{\overline{\text { ran }} F_{0}}^{2}(\mathbb{D})$ follows as (iii) implies $\operatorname{ran} F_{j} \subseteq \overline{\operatorname{ran}}\left(F_{j-1} \quad \cdots \quad F_{0}\right), j \geq 1$, which in turn implies $\operatorname{ran} F_{j} \subseteq \overline{\operatorname{ran}} F_{0}$.

For the implication (iii) $\Rightarrow$ (vi), note that (iii) implies that $\operatorname{ran} \operatorname{col}\left(F_{j}\right)_{j \geq k} \subset$ $\overline{\operatorname{ran}} T_{F}$ for all $k \geq 1$. But then (vi) follows immediately from Lemma 2.1.

The implications (vi) $\Rightarrow$ (iv) $\Rightarrow$ (v) are trivial.

For (v) to (iii) use Lemma 2.1

We are now ready to give a simple proof for the operator-valued Fejér-Riesz theorem. The original proof is due to Rosenblum [17.

Theorem 3.4 ([17]). Let $Q_{j}: \mathcal{H} \rightarrow \mathcal{H}, j=-m, \ldots, m$, be Hilbert space operators so that $Q(z):=\sum_{j=-m}^{m} Q_{j} z^{j} \geq 0, z \in \mathbb{T}$. Then there exists an outer operator polynomial $P(z)=\sum_{j=0}^{m} P_{j} z^{j}$ with $P_{j} \in \mathbf{L}(\mathcal{H}), j=0, \ldots, m$, so that $Q(z)=$ $P(z)^{*} P(z), z \in \mathbb{T}$. 
Proof. Let

$$
Y=\left(Y_{i j}\right)_{i, j=0}^{m}:=S(m)-S(m-1),
$$

where $S(m-1)$ is viewed as an operator on $\mathcal{H}^{m+1}$ (with last row and column equal to 0). By Corollary 3.2 there exist operators $P_{i}: \mathcal{H} \rightarrow \mathcal{H}$ with $\operatorname{ran} P_{i} \subseteq \overline{\operatorname{ran}} P_{0}$ so that

$$
Y=\left(\begin{array}{c}
P_{m}^{*} \\
\vdots \\
P_{0}^{*}
\end{array}\right)\left(\begin{array}{lll}
P_{m} & \cdots & P_{0}
\end{array}\right)
$$

Put $P(z)=\sum_{j=0}^{m} P_{j} z^{j}, Z_{m}=\left(\begin{array}{lll}z^{m} & \cdots & 1\end{array}\right)^{T}$. Then, since in Proposition 3.1 we have that $A=Q_{0}$ and $B=\operatorname{col}\left(Q_{i}\right)_{i=1}^{m}$, we get that

$$
\begin{aligned}
P(z)^{*} P(z)= & Z_{m}^{*} Y Z_{m} \\
= & z^{* m}\left(\sum_{j=-m}^{-1} Q_{j} z^{j}+Q_{0}+\sum_{j=1}^{m} Q_{j} z^{*(-j)}\right) z^{m}+Z_{m-1}^{*} S(m-1) Z_{m-1} \\
& -\bar{z} Z_{m-1}^{*} S(m-1) z Z_{m-1},
\end{aligned}
$$

where the first term equals $Q(z)$ and last two terms cancel when $z \in \mathbb{T}$.

Finally, in order to see that $P(z)$ is outer, use the equivalence (i) $\Leftrightarrow$ (iv) in Theorem 3.3 and the fact that

$$
S\left(T_{P}^{*} T_{P} ; 0\right)=S\left(T_{Q} ; 0\right)=S(S(m) ; 0)=P_{0}^{*} P_{0} .
$$

Next we will show how Lemma 2.5 leads to the existence of inner-outer factorizations for operator-valued polynomials. Recall that $A \in H_{\mathbf{L}(\mathcal{H}, K)}^{\infty}(\mathbb{T})$ is inner if the multiplication operator $M_{A}: H_{\mathcal{H}}^{2}(\mathbb{T}) \rightarrow H_{\mathcal{K}}^{2}(\mathbb{T})$ with symbol $A$ is a partial isometry.

Theorem 3.5 (Existence of inner-outer factorization). Let $A \in H_{\mathbf{L}(\mathcal{H}, K)}^{\infty}(\mathbb{D})$. Then there exists an outer function $F$ and an inner function $V$, so that $A=V F$.

Proof. Consider the Toeplitz operator $T_{Q}:=T_{A}^{*} T_{A}$. By Corollary 3.2 there exist $F_{j}: \mathcal{H} \rightarrow \overline{\operatorname{ran}} F_{0} \subseteq \mathcal{H}$ so that

$$
S(m)=\left(\begin{array}{ccc}
F_{0}^{*} & \cdots & F_{m}^{*} \\
& \ddots & \vdots \\
& & F_{0}^{*}
\end{array}\right)\left(\begin{array}{ccc}
F_{0} & & \\
\vdots & \ddots & \\
F_{m} & \cdots & F_{0}
\end{array}\right)=: \mathcal{F}(m)^{*} \mathcal{F}(m), \quad m \geq 0 .
$$

Note also that

$$
\mathcal{A}(m)^{*} \mathcal{A}(m):=\left(\begin{array}{ccc}
A_{0}^{*} & \cdots & A_{m}^{*} \\
& \ddots & \vdots \\
& & A_{0}^{*}
\end{array}\right)\left(\begin{array}{ccc}
A_{0} & & \\
\vdots & \ddots & \\
A_{m} & \cdots & A_{0}
\end{array}\right) \leq S(m) .
$$

Consider the sequence of operators

$$
\left(\begin{array}{cc}
\mathcal{F}(m) & 0 \\
0 & 0
\end{array}\right)
$$

acting on $\ell_{\mathcal{H}}^{2}\left(\mathbb{N}_{0}\right)$. As $\|S(m)\| \leq\left\|T_{A}^{*} T_{A}\right\|$, it follows that (3.12) is a bounded sequence of operators, and therefore has a subsequence that converges to $T_{F}$, say, 
in the weak-* topology. But then we must have that

$$
T_{F}=\left(\begin{array}{ccc}
F_{0} & & \\
F_{1} & F_{0} & \\
\vdots & \ddots & \ddots
\end{array}\right) .
$$

Also, $\mathcal{A}(m)$ converges to $T_{A}$ in the weak-* topology. But now (3.11) and $\left(\begin{array}{cc}S(m) & 0 \\ 0 & 0\end{array}\right) \leq T_{A}^{*} T_{A}$ yield that $T_{A}^{*} T_{A} \leq T_{F}^{*} T_{F} \leq T_{A}^{*} T_{A}$. Thus $T_{A}^{*} T_{A}=T_{F}^{*} T_{F}$, or equivalently, $A(z)^{*} A(z)=F(z)^{*} F(z)$ a.e on $\mathbb{T}$, where $F(z)=F_{0}+z F_{1}+\ldots$ As $S\left(T_{F}^{*} T_{F} ; 0\right)=F_{0}^{*} F_{0}$ it follows by Theorem 3.3 that $F$ is outer.

Next, notice that we may write

$$
T_{F}=\left(\begin{array}{cc}
F_{0} & 0 \\
\operatorname{col}\left(F_{j}\right)_{j \geq 1} & T_{F}
\end{array}\right), \quad T_{A}=\left(\begin{array}{cc}
A_{0} & 0 \\
\operatorname{col}\left(A_{j}\right)_{j \geq 1} & T_{A}
\end{array}\right) .
$$

Moreover, since $F$ is outer we have that $\operatorname{ran} \operatorname{col}\left(F_{j}\right)_{j \geq 1} \subset \overline{\operatorname{ran}} T_{F}$. By Lemma 2.5 there exists a unique isometry

$$
\tilde{V}=\left(\begin{array}{cc}
V_{11} & 0 \\
V_{21} & V_{22}
\end{array}\right)
$$

acting on $\overline{\operatorname{ran}} F_{0} \oplus \overline{\operatorname{ran}} T_{F}$ so that

$$
\left(\begin{array}{cc}
A_{0} & 0 \\
\operatorname{col}\left(A_{j}\right)_{j \geq 1} & T_{A}
\end{array}\right)=\left(\begin{array}{cc}
V_{11} & 0 \\
V_{21} & V_{22}
\end{array}\right)\left(\begin{array}{cc}
F_{0} & 0 \\
\operatorname{col}\left(F_{j}\right)_{j \geq 1} & T_{F}
\end{array}\right) .
$$

Since $V_{22}$ is an isometry and satisfies $T_{A}=V_{22} T_{F}$ we obtain by the uniqueness statement in Lemma 2.5 that $\tilde{V}=V_{22}$. But that implies that $\tilde{V}$ must be of the form $\tilde{V}=\left(V_{i-j}\right)_{i, j \geq 0}$ with $V_{k}=0$ for $k<0$. Thus $\tilde{V}=T_{V}$ and $V(z)=$ $V_{0}+z V_{1}+z^{2} V_{2}+\ldots$ is inner.

Next we provide new proofs to some more of the various equivalent characterizations that exist for outer functions (see [18]), and obtain a few new ones as well.

Theorem 3.6. Let $F \in H_{\mathbf{L}(\mathcal{H}, \mathcal{K})}^{\infty}(\mathbb{D})$. Denote the Taylor coefficients of $F$ by $F_{j}$, $j \geq 0$. The following are equivalent:

(i) $F$ is outer.

(ii) For any $z \in \mathbb{D}$ and $G \in H_{\mathbf{L}(\mathcal{L}, \mathcal{K})}^{\infty}(\mathbb{D})$ with $G^{*} G=F^{*} F$ a.e. on $\mathbb{T}$,

$$
G(z)^{*} G(z) \leq F(z)^{*} F(z), z \in \mathbb{D} \text {. }
$$

(iii) There exists $z_{0} \in \mathbb{D}$ such that whenever $G \in H_{\mathbf{L}(\mathcal{L}, \mathcal{K})}^{\infty}(\mathbb{D})$ and $G^{*} G=F^{*} F$ a.e. on $\mathbb{T}$,

$$
G\left(z_{0}\right)^{*} G\left(z_{0}\right) \leq F\left(z_{0}\right)^{*} F\left(z_{0}\right) .
$$

(iv) $G \in H_{\mathbf{L}(\mathcal{L}, \mathcal{K})}^{\infty}(\mathbb{D})$ and $G^{*} G=F^{*} F$ a.e. on $\mathbb{T}$ implies

$$
G_{0}^{*} G_{0} \leq F_{0}^{*} F_{0} .
$$

(v) For some $k \in \mathbb{N}_{0}$ we have that $G \in H_{\mathbf{L}(\mathcal{L}, \mathcal{K})}^{\infty}(\mathbb{D})$ and $G^{*} G=F^{*} F$ a.e. on $\mathbb{T}$ implies $\sum_{i=0}^{l} G_{i}^{*} G_{i} \leq \sum_{i=0}^{l} F_{i}^{*} F_{i}, l=0, \ldots, k$, where $G_{i}$ are the Taylor coefficients of $G$.

(vi) For all $k \in \mathbb{N}_{0}$ we have that $G \in H_{\mathbf{L}(\mathcal{L}, \mathcal{K})}^{\infty}(\mathbb{D})$ and $G^{*} G=F^{*} F$ a.e. on $\mathbb{T}$ implies $\sum_{i=0}^{k} G_{i}^{*} G_{i} \leq \sum_{i=0}^{k} F_{i}^{*} F_{i}$, where $G_{i}$ are the Taylor coefficients of $G$. 
(vii) For some $k \in \mathbb{N}_{0}$ we have that $G \in H_{\mathbf{L}(\mathcal{L}, \mathcal{K})}^{\infty}(\mathbb{D})$ and $G^{*} G=F^{*} F$ a.e. on $\mathbb{T}$ implies

$$
\left(\begin{array}{ccc}
F_{0}^{*} & \cdots & F_{k}^{*} \\
& \ddots & \vdots \\
& & F_{0}^{*}
\end{array}\right)\left(\begin{array}{ccc}
F_{0} & & \\
\vdots & \ddots & \\
F_{k} & \cdots & F_{0}
\end{array}\right) \geq\left(\begin{array}{ccc}
G_{0}^{*} & \cdots & G_{k}^{*} \\
& \ddots & \vdots \\
& & G_{0}^{*}
\end{array}\right)\left(\begin{array}{ccc}
G_{0} & & \\
\vdots & \ddots & \\
G_{k} & \cdots & G_{0}
\end{array}\right)
$$

where $G_{i}$ are the Taylor coefficients of $G$.

(viii) For all $k \in \mathbb{N}_{0}$ we have that $G \in H_{\mathbf{L}(\mathcal{L}, \mathcal{K})}^{\infty}(\mathbb{D})$ and $G^{*} G=F^{*} F$ a.e. on $\mathbb{T}$ implies (3.13).

Proof. For any $G \in H_{\mathbf{L}(\mathcal{L}, \mathcal{K})}^{\infty}(\mathbb{D})$ we have that

$$
S\left(T_{G}^{*} T_{G} ; k\right) \geq\left(\begin{array}{ccc}
G_{0}^{*} & \cdots & G_{k}^{*} \\
& \ddots & \vdots \\
& & G_{0}^{*}
\end{array}\right)\left(\begin{array}{ccc}
G_{0} & & \\
\vdots & \ddots & \\
G_{k} & \cdots & G_{0}
\end{array}\right) .
$$

Combining this observation with Theorem $3.3(\mathrm{v})$ and the fact that $T_{F}^{*} T_{F}=T_{G}^{*} T_{G}$, we immediately obtain the implication (i) $\Rightarrow$ (viii).

The implications (viii) $\Rightarrow$ (iv) $\Rightarrow$ (vii) $\Rightarrow$ (v) $\Rightarrow$ (iv), (viii) $\Rightarrow$ (vi) $\Rightarrow$ (v) and (ii) $\Rightarrow$ (iv) $\Rightarrow$ (iii) are trivial.

For (iv) $\Rightarrow$ (i), let $F=V \tilde{F}$ be an inner-outer factorization of $F$. Then, by Theorem 3.3 we have that

$$
F_{0}^{*} F_{0} \leq S\left(T_{F}^{*} T_{F} ; 0\right)=S\left(T_{\tilde{F}}^{*} T_{\tilde{F}} ; 0\right)=\tilde{F}_{0}^{*} \tilde{F}_{0} .
$$

On the other hand, by (iv) $\tilde{F}_{0}^{*} \tilde{F}_{0} \leq F_{0}^{*} F_{0}$, and thus equality $F_{0}^{*} F_{0}=S\left(T_{F}^{*} T_{F} ; 0\right)$ holds. Again applying Theorem 3.3 gives that $F$ is outer.

For the implication (i) $\Rightarrow$ (ii) fix $z \in \mathbb{D}$ and introduce the Blaschke factor $b_{z}(w)=-\frac{w-z}{1-\bar{z} w}, w \in \mathbb{D}$. Then $F$ is outer if and only if $F \circ b_{z}$ is (use that the composition operators $g \rightarrow g \circ b_{z}$ and $g \rightarrow g \circ b_{z}^{-1}$ are bounded operators on $H_{\mathcal{H}}^{2}(\mathbb{D})$ 7, Theorem 3.6]). Moreover $F(w)^{*} F(w)=G(w)^{*} G(w)$ a.e. on $\mathbb{T}$ if and only if $F\left(b_{z}(w)\right)^{*} F\left(b_{z}(w)\right)=G\left(b_{z}(w)\right)^{*} G\left(b_{z}(w)\right)$ a.e. on $\mathbb{T}$. Since $F \circ b_{z}$ is outer, by (i) $\Rightarrow$ (iv), we have that

$$
F\left(b_{z}(0)\right)^{*} F\left(b_{z}(0)\right) \geq \tilde{G}_{0}^{*} \tilde{G}_{0}
$$

for any $\tilde{G}$ such that $\tilde{G}^{*} \tilde{G}=\left(F \circ b_{z}\right)^{*}\left(F \circ b_{z}\right)$ a.e. on $\mathbb{T}$. Now putting $G=\tilde{G} \circ b_{z}^{-1}$ gives that

$$
F(z)^{*} F(z) \geq G(z)^{*} G(z)
$$

for any $G$ with $G^{*} G=F^{*} F$ a.e. on $\mathbb{T}$. Since $z \in \mathbb{D}$ was arbitrary, the result follows.

As (iv) $\Rightarrow$ (i) holds, it follows that if $F$ satisfies (iii), then $F \circ b_{z_{0}}$ is outer. But then $F$ is outer as well. This proves (iii) $\Rightarrow$ (i).

\section{Multivariate OUter POLYNomials}

With the ideas from the previous section we now present a multivariate operatorvalued version of the Fejér-Riesz lemma. As mere positive semidefiniteness on the $d$-torus does not suffice, an additional condition is required for $Q$ to allow an "outer" factorization. This additional condition on $Q$ is given in terms of Schur complements of $T_{Q}$, the Toeplitz operator on $H_{\mathcal{H}}^{2}\left(\mathbb{D}^{d}\right)$ with symbol $Q$.

In order to state the result precisely we need some additional notation. For $z=\left(z_{1}, \ldots, z_{d}\right) \in \mathbb{T}^{d}$ and $k=\left(k_{1}, \ldots, k_{d}\right) \in \mathbb{Z}^{d}$ define $z^{k}:=z_{1}^{k_{1}} \cdots z_{d}^{k_{d}}$. In this 
case $z^{* k}=\bar{z}^{k}=z^{-k}$. We write 0 for $(0, \ldots, 0)$. For set $A, B \subseteq \mathbb{Z}^{d}$ we denote $A-B=\{a-b: a \in A, b \in B\}$. For matrices labeled by elements of $\mathbb{Z}^{d}$ we fix the ordering as lexicographical. Since this is a total ordering, various results from the first section on Schur complements readily translate to this setting. As before, we use the notation $S\left(T_{Q} ; \Lambda\right)$ (or simply $S(\Lambda)$ when no confusion is likely) to indicate a Schur complement of $T_{Q}$ supported in rows and columns $\Lambda \subseteq \mathbb{N}_{0}^{d}$. In the same manner as when we labeled matrices using elements of $\mathbb{N}_{0}$, we sometimes pad Schur complements with zeros. In this way for example, if $\Lambda_{2} \subseteq \Lambda_{1}$, then $S\left(\Lambda_{1}\right)-S\left(\Lambda_{2}\right)$ makes sense. Finally, we need the projections $\Pi_{K}, K \subseteq \mathbb{N}_{0}^{d}$, on $H_{\mathcal{M}}^{2}\left(\mathbb{D}^{d}\right)$ defined by

$$
\Pi_{K}\left(\sum_{k \in \mathbb{N}_{0}^{d}} h_{k} z^{k}\right)=\sum_{k \in K} h_{k} z^{k} .
$$

Theorem 4.1. Let $K=\prod_{i=1}^{d}\left\{0, \ldots, n_{i}\right\}$ and let $Q_{k}: \mathcal{H} \rightarrow \mathcal{H}, k \in K-K$, be Hilbert space operators so that $Q(z):=\sum_{k \in K-K} Q_{k} z^{k} \geq 0, z \in \mathbb{T}^{d}$. Furthermore, let $n=\left(n_{1}, \ldots, n_{d}\right)$ and let $Z_{K}$ be the column matrix $\left(z^{n-k}\right)_{k \in K}$. The following are equivalent:

(i) there exists an operator polynomial $P(z)=\sum_{k \in K} P_{k} z^{k}$ with $P_{j} \in \mathbf{L}(\mathcal{H})$, $j \in K$, so that $Q(z)=P(z)^{*} P(z), z \in \mathbb{T}^{d}$, and

(ii) The operator

$$
\operatorname{ran}\left(\Pi_{\mathbb{N}_{0}^{d} \backslash K} T_{P} \Pi_{\{n\}}\right) \subseteq \overline{\operatorname{ran}}\left(\Pi_{\mathbb{N}_{0}^{d} \backslash K} T_{P} \Pi_{\mathbb{N}_{0}^{d} \backslash K}\right),
$$

and

$$
\operatorname{ran} P_{k} \subseteq \overline{\operatorname{ran}} P_{0}, \quad k \in K
$$

$$
\text { satisfies } Y:=S(K)-S(K \backslash\{n\})
$$

$$
Z_{K}^{*} Y Z_{K}=Q(z), \quad z \in \mathbb{T}^{d}
$$

Proof. Suppose (ii) holds. By Lemma 2.2 there exist $P_{k} \in \mathbf{L}(\mathcal{H}), k \in K$ such that with $P_{K}=\operatorname{row}\left(P_{k}\right)_{k \in K}, Y=P_{K}^{*} P_{K}$. Defining $P(z)=P_{K} Z_{K}=\sum_{k \in K} P_{k} z^{k}$, we obtain from (4.3) that $P(z)^{*} P(z)=Q(z), z \in \mathbb{T}^{d}$. But then $T_{Q}=T_{P}^{*} T_{P}$. View this factorization of $T_{Q}$ with respect to the decomposition

$$
\operatorname{ran} \Pi_{K \backslash\{n\}} \oplus \operatorname{ran} \Pi_{\{n\}} \oplus \operatorname{ran} \Pi_{\mathbb{N}_{0} \backslash K},
$$

in which respect $T_{P}$ is a $3 \times 3$ lower triangular operator matrix. We are now exactly in the situation of Lemma 2.2 with $T^{*}=\Pi_{\mathbb{N}_{0}^{d} \backslash K} T_{P} \Pi_{\{n\}}, U^{*}=\Pi_{\mathbb{N}_{0}^{d} \backslash K} T_{P} \Pi_{\mathbb{N}_{0}^{d} \backslash K}, Q^{*}$ $=\Pi_{\{n\}} T_{P} \Pi_{K \backslash\{n\}}$, and $S^{*}=\Pi_{\{n\}} T_{P} \Pi_{\{n\}}=P_{0}$. Since (2.5) in Lemma 2.2 holds, we obtain (2.6) of Lemma 2.2, which directly translates into the conditions in (i).

For the converse, assume (i). Again, consider the factorization $T_{Q}=T_{P}^{*} T_{P}$ with $T_{P}$ a lower triangular $3 \times 3$ matrix with respect to the decomposition in (4.4). By the equivalence of (2.5) and (2.6) in Lemma 2.2 we have $Y=P_{K}^{*} P_{K}$, where $P_{K}=$ $\operatorname{row}\left(P_{k}\right)_{k \in K}$. Set $P(z)=P_{K} Z_{K}=\sum_{k \in K} P_{k} z^{k}$. Then $Q(z)=P(z)^{*} P(z), z \in$ $\mathbb{T}^{d}$.

The notion of "outerness" of the factor $P$ is given above in equations (4.1) and (4.2). These conditions reduce in the one-variable case to condition (iii) in Theorem 3.3. Clearly, there are many other, perhaps more natural, ways of generalizing the 
notion of outerness to the multivariable case (see, for example, 4]). For instance, the condition $\overline{\operatorname{ran}} T_{P}=H_{\mathcal{M}}^{2}\left(\mathbb{D}^{d}\right)$ or the condition that

$$
P(z)^{*} P(z) \geq L(z)^{*} L(z), z \in \mathbb{D}^{d},
$$

for all $L(z)$ with $P(z)^{*} P(z)=L(z)^{*} L(z), z \in \mathbb{T}^{d}$, are both options. How all these different notions relate to one another remains to be investigated in future work.

Recall from [10] the following result regarding stable factorization (factorizations in terms of polynomials void of zeros in $\overline{\mathbb{D}}^{2}$ ) of a strictly positive scalar-valued trigonometric polynomial.

Theorem $4.2([10])$. Let $K=\left\{0, \ldots, n_{1}\right\} \times\left\{0, \ldots, n_{2}\right\}$ and let $Q_{k}: \mathcal{H} \rightarrow \mathcal{H}$, $k \in K-K$, be scalar valued so that $Q(z):=\sum_{k \in K-K} Q_{k} z^{k}>0, z \in \mathbb{T}^{2}$. Then there exists a scalar-valued polynomial $P(z)=\sum_{k \in K} P_{k} z^{k}$ so that $Q(z)=|P(z)|^{2}$, $z \in \mathbb{T}^{2}$, and $P(z) \neq 0, z \in \overline{\mathbb{D}}^{2}$, if and only if

$$
\left(\Pi_{K \backslash\left\{\left(n_{1}, n_{2}\right)\right\}} T_{Q^{-1}} \Pi_{K \backslash\left\{\left(n_{1}, n_{2}\right)\right\}}\right)^{-1}
$$

has zero entries in locations $(k, l)$ where $k \in\left\{1, \ldots, n_{1}\right\} \times\{0\}$ and $l \in\{0\} \times$ $\left\{1, \ldots, n_{2}\right\}$.

The conditions in Theorem 4.1 and 4.2 are quite different. The following theorem, which gives necessary conditions on the Schur complement in the form of the existence of a decomposition, relates better to Theorem 4.2, as the condition on the Schur complement implies the necessity of some entries in the Schur complement being zero.

Theorem 4.3. Let $K=\left\{0, \ldots, n_{1}\right\} \times\left\{0, \ldots, n_{2}\right\}$ and let $Q_{k}: \mathcal{H} \rightarrow \mathcal{H}, k \in K-K$, be Hilbert space operators so that $Q(z):=\sum_{k \in K-K} Q_{k} z^{k} \geq 0, z \in \mathbb{T}^{2}$. Put

$$
\begin{aligned}
& S_{1}=S\left(T_{Q} ;\left\{0, \ldots, n_{1}-1\right\} \times\left\{0, \ldots, n_{2}\right\}\right), \\
& S_{2}=S\left(T_{Q} ;\left\{0, \ldots, n_{1}\right\} \times\left\{0, \ldots, n_{2}-1\right\}\right), \\
& S_{0}=S\left(T_{Q} ;\left\{0, \ldots, n_{1}-1\right\} \times\left\{0, \ldots, n_{2}-1\right\}\right) .
\end{aligned}
$$

Suppose that $Q(z)=P(z)^{*} P(z), z \in \mathbb{T}^{2}$, where $P(z)=\sum_{k \in K} P_{k} z^{k}, P_{k}: \mathcal{H} \rightarrow \mathcal{H}$, satisfies

$$
\operatorname{ran} \Pi_{\mathbb{N}_{0}^{2} \backslash \widetilde{K}} T_{P} \Pi_{\widetilde{K}} \subset \overline{\operatorname{ran}} \Pi_{\mathbb{N}_{0}^{2} \backslash \widetilde{K}} T_{P} \Pi_{\mathbb{N}_{0}^{2} \backslash \widetilde{K}},
$$

for $\widetilde{K}=\left\{0, \ldots, n_{1}-1\right\} \times\left\{0, \ldots, n_{2}-1\right\},\left\{0, \ldots, n_{1}-1\right\} \times\left\{0, \ldots, n_{2}\right\},\left\{0, \ldots, n_{1}\right\} \times$ $\left\{0, \ldots, n_{2}-1\right\}, K \backslash\left\{\left(n_{1}, n_{2}\right)\right\}$ and $K$. Then

$$
S\left(T_{Q} ; K \backslash\left\{\left(n_{1}, n_{2}\right)\right\}\right)=S_{1}+S_{2}-S_{0}
$$

and

$$
S\left(T_{Q} ; K\right)=T_{Q}-T_{1}\left(T_{Q}-S_{1}\right) T_{1}^{*}-T_{2}\left(T_{Q}-S_{2}\right) T_{2}^{*}+T_{1} T_{2}\left(T_{Q}-S_{0}\right) T_{2}^{*} T_{1}^{*},
$$

where $T_{i}$ is the Toeplitz operator corresponding to the multiplication operator $M_{i}$ : $H_{\mathcal{H}}^{2}\left(\mathbb{D}^{2}\right) \rightarrow H_{\mathcal{H}}^{2}\left(\mathbb{D}^{2}\right)$ with symbol $m_{i}$, where $m_{i}(z)=z_{i}, i=1,2$.

Conversely, suppose that (4.6) and (4.7) hold. Then there exists an operatorvalued polynomial $P(z)=\sum_{k \in K} P_{k} z^{k}: \mathcal{H} \rightarrow \mathcal{H}$ so that $Q(z)=P(z)^{*} P(z), z \in \mathbb{T}^{2}$ and (4.1) and (4.2) hold. 
Proof. Since $Q(z)=P(z)^{*} P(z), z \in \mathbb{T}^{2}$, we have that $T_{Q}=T_{P}^{*} T_{P}$. Let $\widetilde{K}=$ $\left\{0, \ldots, p_{1}\right\} \times\left\{0, \ldots, p_{2}\right\}$ with $p_{i} \in\left\{n_{i}, n_{i}-1\right\}, i=1,2$, or $\widetilde{K}=K \backslash\left\{\left(n_{1}, n_{2}\right)\right\}$, and view the equation $T_{Q}=T_{P}^{*} T_{P}$ with respect to the decomposition

$$
\operatorname{ran} \Pi_{\widetilde{K}} \oplus \operatorname{ran} \Pi_{\mathbb{N}_{0}^{2} \backslash \widetilde{K}} .
$$

Since (4.5) holds true we have by Lemma 2.1 that

$$
S\left(T_{Q} ; \widetilde{K}\right)=\Pi_{\widetilde{K}} T_{P}^{*} \Pi_{\widetilde{K}} T_{P} \Pi_{\widetilde{K}} .
$$

This now yields expressions for all operators in (4.6) and (4.7) in terms of $P$. It is now straightforward to check that (4.6) and (4.7) hold. For illustration purposes let us write out the equalities in the operators in case that $n_{1}=n_{2}=1$ : here we have that $S_{0}=L_{0}^{*} L_{0}, S_{1}=L_{1}^{*} L_{1}, S_{2}=L_{2}^{*} L_{2}, S\left(T_{Q} ; K \backslash\{(1,1)\}\right)=L_{3}^{*} L_{3}$, $S\left(T_{Q} ; K\right)=L_{4}^{*} L_{4}$, where

$$
\begin{aligned}
& L_{0}=\left(\begin{array}{cccc}
P_{00} & 0 & 0 & 0 \\
0 & 0 & 0 & 0 \\
0 & 0 & 0 & 0 \\
0 & 0 & 0 & 0
\end{array}\right), \quad L_{1}=\left(\begin{array}{cccc}
P_{00} & 0 & 0 & 0 \\
0 & 0 & 0 & 0 \\
P_{10} & 0 & P_{00} & 0 \\
0 & 0 & 0 & 0
\end{array}\right), \\
& L_{2}=\left(\begin{array}{cccc}
P_{00} & 0 & 0 & 0 \\
P_{01} & P_{00} & 0 & 0 \\
0 & 0 & 0 & 0 \\
0 & 0 & 0 & 0
\end{array}\right), \quad L_{3}=\left(\begin{array}{ccccc}
P_{00} & 0 & 0 & 0 \\
P_{01} & P_{00} & 0 & 0 \\
P_{10} & 0 & P_{00} & 0 \\
0 & 0 & 0 & 0
\end{array}\right), \\
& L_{4}=\left(\begin{array}{cccc}
P_{0} & 0 & 0 & 0 \\
P_{01} & P_{00} & 0 & 0 \\
P_{10} & 0 & P_{00} & 0 \\
P_{11} & P_{10} & P_{01} & P_{00}
\end{array}\right),
\end{aligned}
$$

and

$$
Y_{0}:=T_{1} T_{Q} T_{1}^{*}-T_{2} T_{Q} T_{2}^{*}+T_{1} T_{2} T_{Q} T_{2}^{*} T_{1}^{*}=\left(\begin{array}{cccc}
Q_{00} & Q_{01}^{*} & Q_{10}^{*} & Q_{11}^{*} \\
Q_{01} & 0 & Q_{1,-1}^{*} & 0 \\
Q_{10} & Q_{1,-1} & 0 & 0 \\
Q_{11} & 0 & 0 & 0
\end{array}\right)
$$

the operators being restricted to rows and columns indexed by $\{(0,0),(0,1),(1,0)$, $(1,1)\}$, as these contain all the nonzero entries. The operators $T_{1}$ and $T_{2}$ restricted to this part correspond to

$$
\left(\begin{array}{llll}
0 & 0 & 0 & 0 \\
0 & 0 & 0 & 0 \\
I & 0 & 0 & 0 \\
0 & I & 0 & 0
\end{array}\right) \quad \text { and } \quad\left(\begin{array}{cccc}
0 & 0 & 0 & 0 \\
I & 0 & 0 & 0 \\
0 & 0 & 0 & 0 \\
0 & 0 & I & 0
\end{array}\right)
$$

respectively. Formulas (4.6) and (4.7) now follow directly. The computations for the case $n_{1} n_{2}>1$ are similar.

For the converse we apply Theorem 4.1 Using (4.6) and (4.7) we find that $Y$ in Theorem 4.1 equals

$$
Y=Y_{0}-\left(S_{1}-T_{1} S_{1} T_{1}^{*}\right)-\left(S_{2}-T_{2} S_{2} T_{2}^{*}\right)+\left(S_{0}-T_{1} T_{2} S_{0} T_{2}^{*} T_{1}^{*}\right)
$$


yielding that

$$
\begin{aligned}
Z_{K}^{*} Y Z_{K}= & Q(z)-\left(1-\left|z_{1}\right|^{2}\right)\left(Z_{K}^{*} S_{1} Z_{K}\right)-\left(1-\left|z_{2}\right|^{*}\right)\left(Z_{K}^{*} S_{2} Z_{K}\right) \\
& +\left(1-\left|z_{1} z_{2}\right|^{2}\right)\left(Z_{K}^{*} S_{0} Z_{K}\right) .
\end{aligned}
$$

Thus for $\left(z_{1}, z_{2}\right) \in \mathbb{T}^{2}$ we obtain equality (4.3). The conclusion now follows from Theorem 4.1 .

Note that Theorem 4.3 is not an if and only if statement due to the different "outerness" requirements on $P$ : in one direction the outerness requirement is (4.5) while in the other direction it is (4.1) and (4.2). We suspect that these two outerness requirements are different, though we have not constructed an example showing this.

Note too that (4.6) implies that $S\left(T_{Q} ; K \backslash\left\{\left(n_{1}, n_{2}\right)\right\}\right)$ has zeros in locations $(k, l)$, where $k \in\left\{1, \ldots, n_{1}\right\} \times\{0\}$ and $l \in\{0\} \times\left\{1, \ldots, n_{2}\right\}$.

\section{REFERENCES}

[1] Gr. Arsene, Zoia Ceauşescu, and T. Constantinescu. Schur analysis of some completion problems. Linear Algebra Appl., 109:1-35, 1988. MR0961563 (89k:47010)

[2] Mihály Bakonyi and Hugo J. Woerdeman. The central method for positive semi-definite, contractive and strong Parrott type completion problems. In Operator theory and complex analysis (Sapporo, 1991), volume 59 of Oper. Theory Adv. Appl., pages 78-95. Birkhäuser, Basel, 1992. MR 1246810 (94j:47024)

[3] A. P. Calderón and R. Pepinsky. On the phases of Fourier coefficients for positive real periodic functions. In Ray Pepinsky, editor, Computing methods and the phase problem in X-ray crystal analysis, pages 339-348. The X-Ray Crystal Analysis Laboratory, Department of Physics, The Pennsylvannia State College, 1952.

[4] Raymond Cheng. Weakly and strongly outer functions on the bidisc. Michigan Math. J., 39(1):99-109, 1992. MR.1137892 (93e:32004)

[5] M. D. Choi, T. Y. Lam, and B. Reznick. Sums of squares of real polynomials. In $K$-theory and algebraic geometry: connections with quadratic forms and division algebras (Santa Barbara, CA, 1992), volume 58 of Proc. Sympos. Pure Math., pages 103-126. Amer. Math. Soc., Providence, RI, 1995. MR1327293 (96f:11058)

[6] T. Constantinescu. Schur analysis of positive block-matrices. In I. Schur methods in operator theory and signal processing, volume 18 of Oper. Theory Adv. Appl., pages 191-206. Birkhäuser, Basel, 1986. MF0902605 (89a:47027)

[7] Carl C. Cowen and Barbara D. MacCluer. Composition operators on spaces of analytic functions. Studies in Advanced Mathematics. CRC Press, Boca Raton, FL, 1995. MR1397026 (97i:47056)

[8] Michael A. Dritschel. On factorization of trigonometric polynomials. Integral Equations Operator Theory, 49:11-42, 2004. MR2057766

[9] Ciprian Foias and Arthur E. Frazho. The commutant lifting approach to interpolation problems, volume 44 of Operator Theory: Advances and Applications. Birkhäuser Verlag, Basel, 1990. MR 1120546 (92k:47033)

[10] Jeffrey S. Geronimo and Hugo J. Woerdeman. Positive extensions, Fejér-Riesz factorization and autoregressive filters in two variables. Ann. of Math. (2), 160:839-906, 2004.

[11] I. C. Gohberg. The factorization problem for operator functions. Izv. Akad. Nauk SSSR Ser. Mat., 28:1055-1082, 1964. MR0174994 (30:5182)

[12] Henry Helson. Lectures on invariant subspaces. Academic Press, New York, 1964. MR0171178 $(30: 1409)$

[13] J. W. McLean and H. J. Woerdeman. Spectral factorizations and sums of squares representations via semidefinite programming. SIAM J. Matrix Anal. Appl., 23(3):646-655 (electronic), 2001/02. MR1896811 (2002k:90074)

[14] Pablo A. Parrilo. Structured semidefinite programs and semialgebraic geometry methods in robustness and optimization. Ph.D. thesis, California Institute of Technology, 2000. 
[15] Frigyes Riesz and Béla Sz.-Nagy. Functional analysis. Dover Books on Advanced Mathematics. Dover Publications Inc., New York, 1990. Translated from the second French edition by Leo F. Boron, Reprint of the 1955 original. MF 1068530 (91g:00002)

[16] Murray Rosenblatt. A multi-dimensional prediction problem. Ark. Mat., 3:407-424, 1958. MR 0092332(19:1098c)

[17] Marvin Rosenblum. Vectorial Toeplitz operators and the Fejér-Riesz theorem. J. Math. Anal. Appl., 23:139-147, 1968. MR0227794 (37:3378)

[18] Marvin Rosenblum and James Rovnyak. Hardy classes and operator theory. The Clarendon Press Oxford University Press, New York, 1985. Oxford Science Publications. MR0822228 (87e:47001)

[19] Walter Rudin. The extension problem for positive-definite functions. Illinois J. Math., 7:532539, 1963. MR0151796 (27:1779)

School of Mathematics and Statistics, Merz Court, University of Newcastle upon Tyne, Newcastle upon Tyne, NE1 7RU, United Kingdom

E-mail address: m.a.dritschel@newcastle.ac.uk

Department of Mathematics, The College of William \& Mary, Williamsburg, Virginia 23185-8795 - and - Department of Mathematics, Katholieke Universiteit Leuven, Celestijnenlaan 200B, B3001 Heverlee, Belgium

Current address: Department of Mathematics, Drexel University, Philadelphia, Pennsylvania 19104

E-mail address: hugo@math.drexel.edu 